\section{Die Medikalisierung weiblicher Biographien im mittleren Alter}

\author{
P. Kolip, G. Glaeske
}

\begin{abstract}
Die Verordnung von Arzneimitteln für Frauen ist nicht losgelöst von einer historischen Entwicklung zu betrachten, die zu einer Medikalisierung körperlicher Umbruchphasen geführt hat. Der Begriff "Medikalisierung" bezieht sich nicht nur auf die Verschreibung von Medikamenten, sondern meint einen Prozess, in dem sich die Medizin für die Begleitung in (körperlichen) Umbruchphasen zuständig erklärt, bzw. - als Kehrseite der Medaille - bei dem die Verantwortung für normale Körperprozesse in die Hände der Medizin gegeben werden.
\end{abstract}

\section{Medikalisierung körperlicher Umbruchphasen}

Die Frauengesundheitsforschung hat in den vergangenen Jahren aufgezeigt, dass kaum eine weibliche Umbruchphase ohne medizinische Begleitung abläuft $[1,2]$. Gut dokumentiert ist diese Entwicklung für die Betreuung von Schwangerschaft und Geburt, die sich von einer von Hebammen geleiteten Geburtshilfe zur Geburtsmedizin gewandelt hat [3, 4]. Die Medikalisierung lässt sich aber auch für andere Lebensphasen aufzeigen. Frauen im mittleren Lebensalter sind hiervon vor allem in zwei Bereichen betroffen: bei der Definition der Wechseljahre als "Hormonmangelkrankheit» [5] und der daraus resultierenden Verschreibung von Hormonpräparaten zur Prävention von Osteoporose, Herzkreislaufkrankheiten und der Alzheimer'schen Erkrankung ohne empirische Evidenz ${ }^{1}$ sowie bei der im folgenden im Vordergrund stehenden Verordnung von psychotropen Substanzen, die häufig vor dem Hintergrund der vermeintlich inadäquaten Bewältigung von Lebenskrisen eingesetzt werden.

Korrespondenz:

Prof. Dr. Petra Kolip

Universität Bremen

Fachbereich 11/BIPS

Postfach 330440

D-28334 Bremen

E-Mail: kolip@bips.uni-bremen.de

Prof. Dr. Gerd Glaeske

Universität Bremen

Fachbereich 11/Zentrum für Sozialpolitik

Parkallee 39

D-28209 Bremen

E-Mail: gglaeske@zes.uni-bremen.de

\section{Verordnung von psychotropen Substanzen}

Der Befund, dass Frauen häufiger psychotrope Substanzen verschrieben bekommen, wurde bereits Anfang der 80er Jahre als differentielle Reaktion des Medizinsystems auf männliche und weibliche Beschwerden beschrieben [7-9]. Auch heute noch lässt sich aufzeigen, dass Frauen psychisch wirkende Arzneimittel häufiger als Männer verschrieben bekommen [10, 11]. Dies könnte zu der Formel verleiten, Männer litten an ihrem Körper, Frauen an ihrer Psyche. Das geschlechtsspezifisch diagnostizierte Krankheitsspektrum legt diese Gleichung nahe: Den auffällig häufigen "männlichen» Diagnosen: Herzinfarkt, Magen-Darm-Ulzera ${ }^{2}$, Leberzirrhose oder Lungenkarzinom $^{3}$ stehen als häufigste weibliche Diagnosen Neurosen, Organneurosen, psychogene Störungen, vegetative Dystonie, Nervosität, Schwäche, Depressivität und Schlafstörungen gegenüber. Da sich diese Verteilung aber nicht mit der "wahren" Prävalenz von Krankheit in Einklang bringen lässt, werden drei wesentliche Hypothesen für die Erläuterung dieser Unterschiede herangezogen [13]:

- Die "women are expressive»-Hypothese, nach der Frauen eine höhere Sensibilität und Emotionalität gegenüber Krankheiten haben, daher häufiger eine Ärztin oder einen Arzt aufsuchen [14, 15], die/der bei bestimmten Belastungssymptomen keinen anderen Rat weiss, als psychotrop wirkende Mittel zu verordnen.

- Die "Substitutionshypothese», nach der Frauen Belastungen anders als Männer bewältigen: Stress, Angst und Alltagsprobleme werden von Frauen eher mit Medikamenten, von Männern eher mit Alkohol «heruntergeschluckt».

- Die «Konvergenzhypothese», nach der der Medikamentenkonsum erst im Rahmen einer Berufstätigkeit sinkt, dann aber Alkohol zur Bewältigung arbeitsplatzbedingter Probleme genutzt wird («männlich dominierte Kultur»).

Der grösste Teil der für Frauen verschriebenen Psychopharmaka und Schlafmittel kommt aus der Familie der Benzodiazepine, einer Arzneimittelgruppe, die nach wie vor in der Therapie - kurzfristig eingesetzt - unverzichtbar ist (z.B. bei Angst- und Panikattacken, präoperativer Muskelrelaxierung, Schlafstörungen usw.). Frauen bekommen insgesamt zwei Drittel dieser beruhigenden Psychopharmaka verordnet und werden daher besonders häufig abhängig von diesen Mitteln, da sich bereits nach 4- bis 6wöchiger Dauertherapie Gewöhnung und nach einigen Monaten Abhängigkeit entwickeln kann.

\footnotetext{
Für einen Überblick siehe [6].

2 Hierauf weisen Verordnungsstatistiken pharmazeutischer Hersteller bezüglich der Anwendung entsprechender Arzneimittel hin, übrigens auch mit Blick auf die Helicobacter pylori-orientierte Eradikationstherapie, die bei Männern häufiger als bei Frauen verordnet wird.

Mit Ausnahme der Magen-Darm-Ulzera gehören die genannten Ereignisse oder Krankheiten auch zu den häufigsten Todesursachen bei Männern im Alter unter 65 Jahren; siehe auch [12].
} 
Diese Benzodiazepinabhängigkeit findet zumeist im Rahmen einer "low-dose-dependency" statt, also einer Niedrigdosisabhängigkeit, die bei einer «normalen» therapeutischen Dosierung über Jahre, z.T. über Jahrzehnte, konstant bleibt - zu einem grossen Teil also eine iatrogen gebahnte und "tolerierte" Abhängigkeit ist. Beim Absetzen der Mittel käme es unmittelbar zu Entzugserscheinungen, damit wird die Dauerverordnung und -einnahme auch zur «Entzugsvermeidungsstrategie» [16].

Das Abhängigkeitspotential steigt mit dem Alter an: Sind es in der Altersgruppe der 40- bis 49jährigen 11,2\% der Frauen und 10,0\% der Männer, die gefährdet sind, eine Abhängigkeitserkrankung durch Tranquilizer zu erleiden, beträgt der Anteil bei den 60- bis 69jährigen bereits 27,0\% bei den Frauen und 20,3 \% bei den Männern [17]. Auffällig ist dabei, dass vor allem in den höheren Altersgruppen als Schlafmittel vor allem solche Benzodiazepine zur Anwendung kommen, die wegen ihrer langen Halbwertzeit insbesondere "hang-over"-Effekte und damit Reaktionsbeeinträchtigung und Sturzgefahr mit dem Risiko schlecht heilender Brüche am Oberschenkel oder an der Hüfte am nächsten Tag verursachen können $[18,19]$.

Rund 65 Prozent aller Benzodiazepinverordnungen werden in Deutschland von Praktikern/-innen und Allgemeinmedizinern/-innen verordnet, 23 Prozent von Internisten/-innen und nur 10 Prozent von Nervenärzten/-ärztinnen. Der allgemeinmedizinische Bereich ist offensichtlich besonders "anfällig» für die unspezifische Verschreibung dieser Substanzen. Die Verordnungsmengen sind zwar in den vergangenen Jahren gesunken, Anlass zur Beruhigung ist dies jedoch noch nicht [20].

\section{Akteurinnen und Akteure im Medikalisierungs- prozess und ihre Motive}

An der Medikalisierung weiblicher Umbruchphasen sind zahlreiche Akteurinnen und Akteure mit jeweils unterschiedlichen Motiven beteiligt. Im Zentrum der Betrachtung sollen im folgenden die drei wesentlichsten Akteurinnen und Akteure stehen: die pharmazeutische Industrie, die Ärztinnen und Ärzte sowie die Frauen als Patientinnen. Zwischen diesen lässt sich eine komplexe Interaktion ausmachen. Um Missverständnissen vorzubeugen: Im folgenden geht es nicht um Medikamentenverordnungen, deren Wirksamkeit bei klar umschriebener Indikation empirisch belegt ist. Vielmehr geht es um die Vielzahl von Verordnungen, deren Sinn zweifelhaft ist, weil sie z.B. eine Lösung für ausserhalb der medizinischen Kompetenz liegende Probleme darstellen. In vielen Fällen sind Medikamente - dies gilt auch für psychotrope Substanzen - eine sinnvolle Intervention, in anderen Fällen sind sie es aber leider nicht, weil die Risiken den Nutzen bei weitem übersteigen, oder weil eine medizinische Intervention weniger sinnvoll ist als z.B. eine psychotherapeutische (z.B. bei der Bewältigung von Lebenskrisen).
Pharmazeutische Industrie

Die Motive der pharmazeutischen Industrie sind schnell benannt: Ihr geht es um die Sicherung bestehender und die Erschliessung neuer Absatzmärkte. In Deutschland nehmen Psychopharmaka Rang 6 bei den verordnungsstärksten Indikationsgruppen ein [21] (Jahresumsatz $2000 \mathrm{zu}$ Lasten der Gesetzlichen Krankenversicherung, d.h. ohne Selbstmedikation: DM 2039,1 Mio. [etwa Fr. 1,7 Mrd.]). Die pharmazeutische Industrie bedient sich vielfältiger Marketinginstrumente; u. a. werden niedergelassene Ärztinnen und Ärzte im Rahmen der Besuche durch Pharmareferenten/-innen vom Nutzen der Medikamente überzeugt [22, 23]. Die Pharmaindustrie nutzt dabei geschickt das Informationsdefizit der Ärztinnen und Ärzte aus (s. u.).

\section{Ärztinnen und Ärzte}

Die Motive der Ärztinnen und Ärzte sind vielfältig. Zunächst einmal ist davon auszugehen, dass sie den Patientinnen helfen wollen und dazu auf das Repertoire zurückgreifen, das sie sich im Rahmen der Ausbildung und praktischen Tätigkeit angeeignet haben. Allerdings lassen sich zahlreiche Hürden benennen, die eine Behandlung nach den Grundlagen der Evidence-based Medicine erschweren. So hat sich die Halbwertszeit des medizinischen Wissens beständig verkürzt, so dass es für den einzelnen Arzt, die einzelne Ärztin kaum noch leistbar ist, angesichts der Informationsflut ständig auf dem aktuellen Stand des Wissens zu sein. Viele Ärztinnen und Ärzte sehen sich aufgrund zeitlicher Restriktionen kaum in der Lage, zu allen für sie relevanten Krankheitsbildern andere Informationen als die der pharmazeutischen Industrie zu suchen, um zu einem unabhängigen Urteil über die Wirksamkeit von Interventionen zu kommen.

Geschlechterstereotype, also Vorstellungen darüber, welche Beschwerden und Bewältigungsstrategien bei Frauen typisch sind, spielen ebenfalls in die Verordnungen hinein. Die psychologische und soziologische Forschung hat gezeigt, dass das Geschlecht eine zentrale Variable ist, die unsere Interaktionen tiefgreifend prägt: Wir verhalten uns - ob wir das wollen oder nicht - unterschiedlich, je nachdem, ob wir es mit einem Mann oder einer Frau zu tun haben [24]. Dies gilt selbstverständlich auch für die Interaktion in der Arztpraxis [25, 26]. Trotz vergleichbarer Beschwerden werden Frauen anders behandelt als Männer - auch weil sie anders über ihre Beschwerden reden. Dies gilt selbst für eindeutig somatische Krankheiten wie den Myokardinfarkt [27-32]. Auch ökonomische Zwänge spielen eine Rolle beim Griff zum Rezeptblock: Frauen, die regelmässig die Praxis aufsuchen, um eine Verordnung $\mathrm{zu}$ erhalten, sind "gute Kundinnen». Und schliesslich sind zeitliche Aspekte relevant, denn ein ausführliches Gespräch z.B. über die psychosoziale Belastung, ist wesentlich aufwendiger als eine Arzneiverordnung.

Die Verkaufsstrategien pharmazeutischer Firmen beeinflussen das ärztliche Verordnungsverhalten zwar massiv, doch das Angebot allein erzwingt nicht die Anwendung der Mittel. Ärztinnen und Ärzten sollte 
durchaus abverlangt werden dürfen, dass sie neben den Hochglanzbroschüren pharmazeutischer Hersteller auch vergleichende Bewertungen industrieunabhängiger Institutionen berücksichtigen. Eine sorglose Verschreibungspraxis ist typisch für wenig oder nur einseitig informierte Ärztinnen und Ärzte. Nur knapp 10 Prozent aller Ärztinnen und Ärzte verordnen bereits 50 Prozent der gesamten Menge der obengenannten Schlaf- und Beruhigungsmittel. Eine relativ kleine Gruppe von Ärztinnen und Ärzten in «Benzodiazepinschwerpunktpraxen" ist also verantwortlich für die "Sucht auf Rezept». In der Zwischenzeit sind auch andere Arzneimittelgruppen hinzugekommen, Verordnungen aus dem Bereich der niedrig dosierten Neuroleptika oder der selektiven Serotonin-Wiederaufnahmehemmer (SSRI) übernehmen zum Teil den Platz der Benzodiazepinderivate. Diese Mittel machen zwar nicht abhängig, sind aber genauso fehl plaziert, wenn sie nicht indikationsgerecht eingesetzt werden. Ausserdem sind bei diesen Gruppen andere unerwünschte Wirkungen zu beachten - bei den niedrig dosierten Neuroleptika zum Beispiel sind nämlich quälende extrapyramidalmotorische Störungen und Dyskinesien keineswegs ausgeschlossen.

\section{Frauen als Patientinnen}

Frauen sind nicht nur Opfer der Medikalisierung, in vielen Fällen fordern sie diese aktiv ein, weil sie mit dem Wunsch nach einer Arzneimittelverordnung in die Praxis kommen. Die Motive hierfür sind vielfältig: Zunächst einmal wünschen sich die Frauen eine Linderung ihrer Beschwerden und sie versprechen sich von den psychotropen Substanzen eine Lösung ihrer Probleme, die nur vor dem Hintergrund der geschlechtlichen Arbeitsteilung, die Männern und Frauen unterschiedliche soziale Rollen zuweist, zu verstehen sind. Nach wie vor sind es überwiegend die Frauen, die für die Haushalts- und Familienarbeit zuständig sind [33], und die Alltagsrealität von Frauen, ob sie berufstätig sind oder nicht, ist oft geprägt durch die widersprüchlichen Anforderungen der Arbeitswelt und der Familie [34-36]. In der Arbeitswelt muss Privates weitgehend ausgeblendet werden. Im Binnenraum Familie wird es - trotz Gleichstellungs- und Partnerschaftsrhetorik - immer noch primär als Aufgabe der Frauen angesehen, ein warmes und vertrauensvolles Klima herzustellen, das die Härten der äusseren Welt auszugleichen hilft und dem Partner die Möglichkeit zur Regeneration schafft.

Die besondere Überlastung vieler Frauen entsteht im Versuch, diesen Balanceakt fertigzubringen: einerseits in der Berufswelt zu funktionieren und andererseits in der Familie Spannungen auszugleichen, Konflikte abzupuffern, Beistand zu leisten, Mut zu machen, Stütze zu sein und generell Harmonie herzustellen. Zur widersprüchlichen Belastung durch Haushalt, Berufstätigkeit und Kindererziehung kommt meist noch der Anspruch hinzu, dem Image der jungdynamischen und sexuell attraktiven Frau zu entsprechen. Daneben kommt jedoch in den letzten Jahren immer mehr zum Tragen, dass in Zeiten hoher Arbeitslosigkeit vor allem Frauen ohne Beschäfti- gung sind. Die Studie von Gmel [37] konnte zeigen, dass die fehlende Erwerbstätigkeit einen erhöhten Konsum von Schlaf- und Beruhigungsmitteln nach sich zieht, wobei sich dieses Problem ab einem Alter von 45 Jahren verschärft. Hier beginnt, bedingt durch den Auszug allfälliger Kinder aus dem Haushalt, der zwar vielfach belastende, aber dennoch protektive und in seiner Funktionsanforderung stabilisierende Faktor "Familie» seine Wirkung zu verlieren. Bezahlte Erwerbstätigkeit bekommt daher für Frauen insbesondere im mittleren Lebensabschnitt eine entscheidende Bedeutung im Hinblick auf den Medikamentenkonsum und auf eine möglicherweise drohende Abhängigkeit. "Aus präventiver Sicht», so das Fazit der schweizerischen Untersuchung, «bleibt $\mathrm{zu}$ fordern, dass Frauen einerseits ein besserer gleichberechtigter Zugang in die Erwerbstätigkeit ermöglicht wird. Zum anderen aber sind gerade Beschäftigungsmöglichkeiten für Frauen ab 45 Jahren zu schaffen. Dadurch würden Möglichkeiten geboten, um die einsetzende Leere in der Zeit nach der Kinderbetreuung aufzufangen. Es ist jedoch zu befürchten, dass dies ein (frommer Wunsch) bleibt. Die Arbeitslosenrate liegt mit etwa 4,5\% in der Schweiz zwar deutlich unter derjenigen anderer westlicher Nationen [wohl wahr, in Deutschland über 10\%, d.V.], doch sie ist seit Mitte der 70er Jahre ständig im Steigen begriffen und betrifft neben Ausländern in erster Linie Frauen. [...] Erwerbstätigkeit hat einen so entscheidenden Einfluss auf die Einnahme von Schlaf- und Beruhigungsmitteln, dass Frauen wegen der Ungleichstellung im Erwerbsleben und vermutlich auch durch die ungleiche Verteilung der zu leistenden Familien- und Beziehungsarbeit deutlich benachteiligt sind."

Auch die von Geschlechtsstereotypen geprägte Arzt-Patientin-Interaktion, an der Ärztinnen/Ärzte und Patientinnen gleichermassen beteiligt sind, begünstigt offenbar eine Medikalisierung. Die Art, wie Frauen über ihre Probleme sprechen, indem sie z.B. Zusammenhänge zu ihrer Lebenssituation herstellen, trägt dazu bei, dass bei ihnen häufiger als bei Männern nach psychosomatischen Anteilen gesucht wird, die eine Verordnung von Psychopharmaka rechtfertigen könnten. Mit dem Aufsuchen einer ärztlichen Praxis geben sie darüber hinaus Verantwortung ab, indem sie dem Problem eine körperliche Ursache zuordnen und die Medizin als zuständig für die Lösung erklären. Hintergrund hierfür ist die "medizinische Sozialisation" von Frauen, d.h. die Erfahrung, dass körperliche und psychische Umbruchphasen in die Hände der Medizin gehören. Eine medizinische Diagnose wirkt darüber hinaus entlastend: Sie können ihr Problem benennen und haben durch die Einnahme von Medikamenten das Gefühl, etwas für die Genesung zu tun. Die Einnahme von Medikamenten ist dabei weniger aufwendig als z.B. eine Psychotherapie oder die Änderung des Lebensstils. Risiken werden aber oftmals übersehen: Im Gegensatz zu weiblichen Umbruchphasen ist die Nebenwirkung Abhängigkeit nach der unkritischen Verordnung von bestimmten psychotropen Arzneimitteln eine wirkliche Krankheit! 


\section{Literatur}

1 Kolip P (Hrsg.). Weiblichkeit ist keine Krankheit. Die Medikalisierung körperlicher Umbruchphasen im Leben von Frauen. Weinheim: Juventa; 2000.

2 Honegger C. Überlegungen zur Medikalisierung des weiblichen Körpers. In: Imhof A (Hrsg.). Leib und Leben in der Geschichte der Neuzeit. Vorträge eines internationalen Colloquiums, Berlin: Duncker \& Humblot-Verlag; 1983. S. 203-14.

3 Huerkamp C. Ärzte und Professionalisierung in Deutschland. Überlegungen zum Wandel des Arztberufes im 19. Jahrhundert. Geschichte und Gesellschaft 1980;8:349-82.

4 Ellerbrock D. Geschlecht, Gesundheit und Krankheit in historischer Perspektive. In Hurrelmann K, Kolip P. (Hrsg.). Geschlecht - Gesundheit - Krankheit. Männer und Frauen im Vergleich. Bern: Hans Huber; 2002 (im Druck).

5 Panke-Kochinke B. Die Wechseljahre der Frau. Aktualität und Geschichte 1772-1996. Opladen: Leske + Budrich; 1998.

6 Beckermann MJ. Evaluation epidemiologischer Studien zur Östrogen-Gestagen-Hormontherapie. Schweiz Med Forum 2001;1(5):91-119 (www.medicalforum.ch/pdf_d/2001/ 2001-05/2001-05-398.pdf).

7 Vogt I. Für alle Leiden gibt es eine Pille. Opladen: Westdeutscher Verlag; 1985.

8 Glaeske G. Zur Epidemiologie und zum Abhängigkeitspotential von Tranquilizern und anderen Psychopharmaka. In: Deutsche Hauptstelle gegen die Suchtgefahren (Hrsg.). Jahrbuch 1987 zur Frage der Suchtgefahren. Geesthacht: Neuland; 1987. S. 111-37.

9 Krause-Girth C. Schein-Lösungen. Die Verschreibungspraxis von Psychopharmaka. Bonn: Psychiatrie-Verlag; 1989.

10 Simoni-Wastila L. Gender and psychotropic drug use. Med Care 1998;36:88-94.

11 Glaeske G, Janhsen K. GEK-Arzneimittel-Report. Sankt Augustin: Asgard; 2001.

12 Courtenay WH. Constructions of masculinity and their influence on men's well-being: a theory of gender and health. Soc Sci Med 2000;50:1385-401.

13 Franke A, Elsesser K, Sitzler F, Algermissen G, Kötter S. Gesundheit und Abhängigkeit bei Frauen: Eine salutogenetische Verlaufsstudie. Cloppenburg: Runge; 1998.

14 Gijsbers van Wijk CM, van Vliet KP, Koltz AM, Everaed WT. Symptom sensitivity and sex differences: a review of health surveys in the United States and the Netherlands. Women and Health 1991;17:109-38.

15 Sayer GP, Britt H. Sex differences in prescribed medications: Another case of discrimination in general practice. Soc Sci Med 1997;45:1581-7.

16 Glaeske G. Psychotrope und andere Arzneimittel mit Missbrauchs- und Abhängigkeitspotenzial. In: Deutsche Hauptstelle gegen die Suchtgefahren (Hrsg.): Jahrbuch Sucht 2001. Geesthacht: Neuland; 2000. S. 63-79.

17 Remien J. Bestimmung der Arzneimittelabhängigkeit durch eine quantitative Analyse des individuellen Verbrauchs aller ärztlich verordneten Arzneimittel. Bergisch Gladbach: IKK-Bundesverband; 1994. Projektbericht.
18 Weyerer S. Medikamentensucht im Alter. Reader zur Anhörung "Sucht auf Rezept" der Grünen im Bayrischen Landtag am 26. Januar 2001. München; 2001. S. 20-7.

19 Wang PS, Bohn RL, Glynn RJ, Mogun H, Avom J. Hazardous benzodiazepine regimens in the elderly: effects of half-life, dosage, and duration on risk of hip fracture. Am J Psychiatry 2001;158:892-8.

20 Faber U. Tranquilizer: Kein Anlass zur Beruhigung. In: Faber U, Glaeske G, Puteanus U, Schubert I (Hrsg.). Wechselwirkungen - Beiträge zu Pharmazie und Politik. Frankfurt: Mabuse; 1999. S. 157-63.

21 Schwabe U, Paffrath D (Hrsg.). Arzneiverordnungs-Report 2001. Berlin: Springer; 2001.

22 von Seemann N. Planung des persönlichen Vertriebs im Pharmamarketing. Bern: Peter Lang; 1995.

23 Becker HE. Kommunikations-Strategien im Pharma-Markt. Heidelberg: Physica-Verlag; 1992.

24 West C, Zimmerman D. Doing gender. Gender Society 1987; 1:125-51.

25 Hall JA, Roter DL. Patient gender and communication with physicians: Results of a community-based study. Women's Health: Research on Gender, Behavior, and Policy 1995; 1:77-95.

26 Roter DL, Hall JA. Gender differences in patient-physician communication. In Gallant SJ, Keita GP, Rayak-Schaler R. (eds.). Health care for women. Psychological, social, and behavioral influences. Washington: American Psychological Association; 1997. p. 57-71.

27 Wenger NK, Speroff L, Packard B. Cardiovascular health and disease in women. N Engl J Med 1993;329:247-56.

28 Swahn E. The care of patients with ischaemic heart disease from a gender perspective. Eur Heart J 1998;19:1758-65.

29 Vaccarino V, Parsons L, Every NR, Barron HV, Krumholz HM. Sex-based differences in early mortality after myocardial infarction. N Engl J Med 1999;341:217-24.

30 Murabito JM. Women and cardiovascular disease: Contributions from the Framingham Heart Study. J Am Med Women's Ass 1995;50:35-9.

31 Healy B. The Yentl syndrome. N Engl J Med 1991;325:274-6.

32 Clarke KW, Gray D, Keating NA, Hampton JR. Do women with acute myocardial infarction receive the same treatments as men? Brit Med J 1994;309:563-6.

33 Künzler J, Walter W, Reichart E, Pfister G. Gender division of labour in unified Germany. Tilburg: University Press; 2001.

34 Beck-Gernsheim E. Das halbierte Leben. Männerwelt Beruf, Frauenwelt Familie. Frankfurt/Main: Fischer; 1980.

35 Beck-Gernsheim E. Arbeitsteilung, Selbstbild und Lebensentwurf. Neue Konfliktlagen in der Familie. Kölner Zeitschrift für Soziologie und Sozialpsychologie 1992;44:273-91.

36 Resch M. Familien- und Erwerbsarbeit. In Hurrelmann K, Kolip P (Hrsg.). Geschlecht - Gesundheit - Krankheit. Männer und Frauen im Vergleich. Bern: Hans Huber; 2002 (im Druck).

37 Gmel G. Konsum von Schlaf- und Beruhigungsmitteln in der Schweiz: Nehmen Frauen mehr Medikamente oder sind mehr Männer erwerbstätig? Zeitschrift für Gesundheitswissenschaften 1997;1:14-31. 